\title{
Shensongyangxin protects against pressure overload-induced cardiac hypertrophy
}

\author{
DI-FEI SHEN ${ }^{1-3^{*}}$, QING-QING WU ${ }^{1,3^{*}}$, JIAN NI $^{1,3}$, WEI DENG ${ }^{1,3}$, CONG WEI $^{4}$, ZHEN-HUA JIA ${ }^{4}$, \\ HENG ZHOU ${ }^{1,3}$, MENG-QIAO ZHOU ${ }^{1,3}$, ZHOU-YAN BIAN ${ }^{1,3}$ and QI-ZHU TANG ${ }^{1,3}$ \\ ${ }^{1}$ Department of Cardiology, Renmin Hospital of Wuhan University, Wuhan, Hubei 430060; \\ ${ }^{2}$ Department of Cardiology, The Fifth Teaching Hospital of Xinjiang Medical University, Xinjiang, \\ Chinese Autonomous Region 830011; ${ }^{3}$ Cardiovascular Research Institute of Wuhan University, Wuhan, Hubei 430060; \\ ${ }^{4}$ The Integration of Traditional and Western Medical Research Academy of Hebei, Shijiazhuang, Hebei 050035, P.R. China
}

Received November 28, 2014; Accepted October 5, 2015

DOI: $10.3892 / \mathrm{mmr} .2015 .4598$

\begin{abstract}
Shensongyangxin (SSYX) is a medicinal herb, which has long been used in traditional Chinese medicine. Various pharmacological activities of SSYX have been identified. However, the role of SSYX in cardiac hypertrophy remains to be fully elucidated. In present study, aortic banding (AB) was performed to induce cardiac hypertrophy in mice. SSYX (520 mg/kg) was administered by daily gavage between 1 and 8 weeks following surgery. The extent of cardiac hypertrophy was then evaluated by pathological and molecular analyses of heart tissue samples. In addition, in vitro experiments were performed to confirm the in vivo results. The data of the present study demonstrated that SSYX prevented the cardiac hypertrophy and fibrosis induced by AB, as assessed by measurements of heart weight and gross heart size, hematoxylin and eosin staining, cross-sectional cardiomyocyte area and the mRNA expression levels of hypertrophic markers. SSYX also inhibited collagen deposition and suppressed the expression of transforming growth factor $\beta$ (TGF $\beta$ ), connective tissue growth factor, fibronectin, collagen I $\alpha$ and collagen III $\alpha$, which was mediated by the inhibition of the TGF $\beta /$ small mothers against decapentaplegic (Smad) signaling pathway. The inhibitory action of SSYX on cardiac hypertrophy was mediated by the inhibition of Akt signaling. In vitro investigations in the rat $\mathrm{H} 9 \mathrm{c} 2$ cardiac cells also demonstrated that SSYX attenuated angiotensin II-induced cardiomyocyte hypertrophy. These findings suggested that SSYX attenuated cardiac hypertrophy and
\end{abstract}

Correspondence to: Professor Qi-Zhu Tang, Department of Cardiology, Renmin Hospital of Wuhan University, 238 Jiefang Road, Wuhan, Hubei 430060, P.R. China

E-mail: qztang@whu.edu.cn

*Contributed equally

Key words: shensongyangxin, cardiac hypertrophy, cardiac fibrosis, Akt, transforming growth factor $\beta /$ small mothers against decapentaplegic fibrosis in the pressure overloaded mouse heart. Therefore, the cardioprotective effect of SSYX is associated with inhibition of the Akt and TGF $\beta /$ Smad signaling pathways.

\section{Introduction}

Cardiac hypertrophy is an adaptive response of the heart to numerous neurohormonal stimuli. Physiological cardiac hypertrophy occurs in response to exercise and results in the adaptation of contractility to the increase in wall stress $(1,2)$, whereas pathological hypertrophy occurs as a response to volume or pressure overload, which is associated with increased interstitial fibrosis, cell death and cardiac dysfunction, ultimately leading to heart failure (3). These progressive changes have long been considered as irreversible (4). Divergent signaling mechanisms may lead to distinct patterns of hypertrophy, although there is a level of overlapping between certain signaling pathways. Notably, the Akt and transforming growth factor $\beta$ (TGF $\beta$ )/small mothers against decapentaplegic (Smad) signaling pathways $(5,6)$ lead to cardiomyocyte hypertrophy and cardiac fibrosis, contributing to pathological hypertrophy and heart failure (HF) (7). However, how to inhibit these signaling pathways in the heart during increased biomechanical stress remains to be fully elucidated.

Shensongyangxin (SSYX), is a traditional Chinese medicine, which was originally developed for the treatment of cardiac tachyarrhythmias, and is a component of the traditional Chinese materia medica, consisting of 12 ingredients, including the plants ginseng, Radix, dogwood, Salvia, Semen (fried), mistletoe, red peony, Eupolyphaga, nard, berberine, kadsura and keel (8). Previous investigations have focussed on the function of SSYX in cardiac arrhythmia, including tachyarrhythmia, bradycardia, paroxysmal atrial fibrillation (AF) and premature ventricular contractions (9-11). In addition, a previous study demonstrated that, when combined with routine pharmacotherapy, SSYX significantly normalizes heart rate variability and heart rate turbulence, and reduces the incidence of ventricular tachycardia and AF, suggesting that SSYX may provide electrophysiological benefits to patients with chronic HF (12). In a coronary artery ligation rat model, SSYX inhibits ventricular remodeling, and improves 
the electrophysiological base material of the heart, which may be associated with affecting the ion channels of myocardial cellular membranes (13). These previous findings suggest that SSYX has a potential protective effects on the cardiovascular system. However, whether SSYX is able to arrest the development of pressure overload-induced cardiac remodeling and prevent HF remains to be elucidated. In the present study, mice were subjected to AB and were treated with SSYX. The current study investigated whether SSYX attenuated cardiac hypertrophy and fibrosis, and aimed to provide experimental evidence for the potential application of SSYX in the treatment of cardiac remodeling and $\mathrm{HF}$.

\section{Materials and methods}

Animals and animal models. All animal procedures were performed in accordance with the Guide for the Care and Use of Laboratory Animals published by the US National Institutes of Health, and were approved by the Animal Care and Use Committee of Renmin Hospital of Wuhan University (Wuhan, China) (14). The surgical procedures and subsequent analyses were performed in a blinded-manner for all groups. Adult male C57/BL6 mice ( $\mathrm{n}=60$; 8-10 weeks old; Beijing HFK Bioscience Co., Ltd., Beijing, China) were used for the present study. Mice were housed with controlled temperature and humidity under a 12-h light/dark cycle with free access to food and water in the Cardiovascular Research Institute of Wuhan University (Wuhan, China). The animals were allowed to acclimatize to the laboratory environment for a minimum of one week, then randomly assigned to one of four groups: Vehicle sham group $(n=15)$; vehicle AB group $(n=15)$; SSYX sham group $(n=15)$; and the SSYX AB group $(n=15)$. Aortic banding $(A B)$ was performed, as previously described (15). In brief, mice were anesthetized with 3\% sodium pentobarbital (Sigma-Aldrich, St. Louis, MO, USA) by intraperitoneal injection. Mice were then orally intubated in a supine position with a heating pad. Artificial respiration was maintained using a rodent ventilator (Somnosuite model; Kent Scientific Corporation, Torrington, CT, USA). The aortic arch branch was exposed with a chest expander upon opening of the second and third intercostals by an incision. The vessel was ligated using a $26 \mathrm{G} / 27 \mathrm{G}$ syringe needle placed parallel above the vessel. Subsequent to rapid withdrawal of the needle to achieve aortic constriction, the chest was closed in layers and a total of $0.1 \mathrm{ml} 0.5 \%$ bupivacaine (Sigma-Aldrich) was injected subcutaneously close to the edges of the skin incision to alleviate postoperative pain. After 1 week of either chronic pressure overload, generated by the $\mathrm{AB}$, or sham surgery (control group), the mice (15/group) received gavage with $0.3 \mathrm{ml}$ saline containing SSYX (520 mg/kg; Shijiazhuang Yiling Pharmaceutical Co., Ltd., Shijiazhuang, China) or normal saline (vehicle) for 7 weeks. The mice were subsequently sacrificed by cervical dislocation, and their hearts and lungs were harvested and weighed in order to compare the heart weight/body weight (HW/BW; mg/g), lung weight/body weight (LW/BW; mg/g) and heart weight/tibia length (HW/TL; $\mathrm{mg} / \mathrm{ml}$ ) ratios in the SSYX-treated and vehicle-treated mice.

Histological analysis. The hearts were arrested in diastole using 10\% KCl (Sinopharm Chemical Reagent Co., Ltd.,
Shanghai, China), and were then weighed, fixed by perfusion with $10 \%$ formalin (Sinopharm Chemical Reagent Co., Ltd.) and embedded in paraffin (Fisher Scientific UK Ltd., Loughborough, England). The hearts were then sectioned transversely, close to the apex, in order to visualize the left and right ventricles. Several sections of each heart (4-5 $\mu \mathrm{m})$ were prepared, stained with hematoxylin and eosin (Baso Diagnostics, Inc., Zhuhai, China) for histopathological analysis, and picrosirius red [PSR; Hyde Venture (Beijing) Biotech Co., Ltd., Beijing, China] for collagen deposition, following which the sections were visualized using light microscopy (Olympus FSX100; Olympus Corporation, Tokyo, Japan). A single myocyte was measured using an image quantitative digital analysis system (Image Pro-Plus 6.0; Media Cybernetics, Inc. Rockville, MD, USA). The outline of 100 myocytes were traced in each group.

Reverse transcription-quantitative polymerase chain reaction $(R T-q P C R)$. RT-qPCR was used to detect the mRNA expression levels of hypertrophic (atrial natriuretic peptide, ANP; brain natriuretic peptide, BNP; $\alpha$-myosin heavy chain, $\alpha$-MHC; and $\beta$-MHC) and fibrotic markers (TGF $\beta 1$; connective tissue growth factor, CTGF; fibronection; collagen I; and collagen III). Total RNA was extracted from the frozen, pulverized mouse cardiac tissue samples using TRIzol ${ }^{\circledR}$ reagent (cat. no. 15596-026; Roche Diagnostics, Basel, Switzerland). The yield and purity levels of the RNA were spectrophotometrically estimated using A260/A280 and A230/A260 ratios, obtained via aSmartSpec Plus Spectrophotometer (Bio-Rad Laboratories, Inc., Hercules, CA, USA). The RNA ( $2 \mathrm{mg}$ of each sample) was reverse transcribed into cDNA using oligo(dT) primers (cat. no.04896866001; Roche Diagnostics) and a Transcriptor First Strand cDNA Synthesis kit (cat. no. 04896866001; Roche Diagnostics). The PCR amplifications were quantified using LightCycler $480 \mathrm{SYBR}^{\circledR}$ Green 1 Master Mix (cat. no. 04707516001; Roche Diagnostics) in the LightCycler $^{\circledR} 480$ Real-Time Quantitative PCR System (Roche Diagnostics). Briefly, subsequent to a 5 min initial denaturation at $95^{\circ} \mathrm{C}$, a total of 42 primer-extension cycles were conducted. Each cycle consisted of a $10 \mathrm{sec}$ denaturation step at $95^{\circ} \mathrm{C}$, a $20 \mathrm{sec}$ annealing step at $60^{\circ} \mathrm{C}$ and a $20 \mathrm{sec}$ incubation at $72^{\circ} \mathrm{C}$ for extension. Then a final extension step was conducted at $72^{\circ} \mathrm{C}$ for $10 \mathrm{~min}$. The double standard curve was used to quantify the PCR results. The results were normalized to the mRNA expression of glyceraldehydes-3-phosphate dehydrogenase (GAPDH). The oligonucleotide primer sequences are listed in Table I.

Western blotting. The cardiac tissue samples were lysed in radioimmunoprecipitation assay lysis buffer (Roche Diagnostics), and protein concentration was measured using a Bicinchoninic Acid Protein Assay kit (cat. no. 23227; Thermo Fisher Scientific, Inc., Waltham, MA, USA) with a Synergy HT ELISA reader (Bio-Tek Instruments, Inc., Winooski, VT, USA). The protein lysates $(50 \mu \mathrm{g})$ were separated on $10 \%$ SDS-PAGE (Wuhan Goodbio Technology Company, Wuhan, China) and transferred onto Immobilon-FL transfer membranes (cat. no. IPFL00010; Merck Millipore, Darmstadt, Germany), and were then blocked with $5 \%$ non-fat milk for $2 \mathrm{~h}$. The following primary antibodies (obtained from Cell Signaling Technology, Inc., Danvers, MA, USA, unless otherwise stated) were used: Rabbit polyclonal GAPDH (cat. no. sc-25778; Santa Cruz Biotechnology, Inc., 
Table I. Primer sequences for reverse transcription-quantitative polymerase chain reaction analysis.

\begin{tabular}{lll}
\hline mRNA & \multicolumn{1}{c}{ Forward primer } & \multicolumn{1}{c}{ Reverse primer } \\
\hline ANP (mouse) & 5'-ACCTGCTAGACCACCTGGAG-3' & 5'-CCTTGGCTGTTATCTTCGGTACCGG-3' \\
BNP (mouse) & 5'-GAGGTCACTCCTATCCTCTGG-3' & 5'-GCCATTTCCTCCGACTTTTCTC-3' \\
$\alpha$-MHC (mouse) & 5'-GTCCAAGTTCCGCAAGGT-3' & 5'-AGGGTCTGCTGGAGAGGTTA-3' \\
$\beta$-MHC (mouse) & 5'-CCGAGTCCCAGGTCAACAA-3' & 5'-CTTCACGGGCACCCTTGGA-3' \\
TGFß1 (mouse) & 5'-ATCCTGTCCAAACTAAGGCTCG-3' & 5'-ACCTCTTTAGCATAGTAGTCCGC-3' \\
CTGF (mouse) & 5'-TGTGTGATGAGCCCAAGGAC-3' & 5'-AGTTGGCTCGCATCATAGTTG-3' \\
Fibronectin (mouse) & 5'-CCGGTGGCTGTCAGTCAGA-3' & 5'-CCGTTCCCACTGCTGATTTATC-3' \\
Collagen I (mouse) & 5'-AGGCTTCAGTGGTTTGGATG-3' & 5'-CACCAACAGCACCATCGTTA-3' \\
Collagen III (mouse) & 5'-CCCAACCCAGAGATCCCATT-3' & 5'-GAAGCACAGGAGCAGGTGTAGA-3' \\
GAPDH (mouse) & 5'-ACTCCACTCACGGCAAATTC-3' & 5'-TCTCCATGGTGGTGAAGACA-3' \\
ANP (rat) & 5'-AAAGCAAACTGAGGGCTCTGCTCG-3' & 5'-TTCGGTACCGGAAGCTGTTG CA-3' \\
BNP (rat) & 5'-CAGCAGCTTCTGCATCGTGGAT-3' & 5'-TTCCTTAATCTGTCGCCGCTGG-3' \\
$\beta$-MHC (rat) & 5'-TCTGGACAGCTCCCCATTCT-3' & 5'-CAAGGCTAACCTGGAGAAGATG-3' \\
GAPDH (rat) & 5'-GACATGCCGCCTGGAGAAAC-3' & 5'-AGCCCAGGATGCCCTTTAGT-3' \\
\hline
\end{tabular}

ANP, atrial natriuretic peptide; BNP, brain natriuretic peptide; MHC, myosin heavy chain; TGF $\beta 1$, transforming growth factor $\beta 1$; CTGF, connective tissue growth factor; GAPDH, glyceraldehydes-3-phosphate dehydrogenase.

Dallas, TX, USA), rabbit monoclonal phosphorylated (p)-Akt (cat. no. 4691), rabbit monoclonal total (T)-Akt (cat. no. 4060), rabbit monoclonal p-glycogen synthase kinase (GSK) $3 \beta$ (cat. no. 9315), rabbit monoclonal T-GSK3 $\beta$ (cat. no. 9322), rabbit polyclonal p-mammalian target of rapamycin (mTOR; cat. no. 2983), rabbit monoclonal T-mTOR (cat. no. 2971), rabbit monoclonal p-4E binding protein 1 (4EBP1; cat. no. 2855P), rabbit monoclonal T-4EBP1 (cat. no. 9644P), rabbit monoclonal p-p70 (cat. no. 9234P), rabbit monoclonal T-p70 (cat. no. 2708), rabbit polyclonal p-forkhead box protein (FOXO)1; cat. no. 9461P), rabbit monoclonal T-FOXO1 (cat. no. 2880P), rabbit polyclonal p-FOXO3a (cat. no. 9465P), rabbit monoclonal T-FOXO3a (cat. no. 2497P) and rabbit polyclonal TGF $\beta$ (cat. no. ab66043; Abcam, Cambridge, UK), mouse monoclonal Smad4 (cat. no. sc-7966; Santa Cruz Biotechnology, Inc.), rabbit monoclonal p-Smad1/5 (cat. no. 9516) and goat polyclonal T-Smad1/5 (cat. no. sc-6201; Santa Cruz Biotechnology, Inc.). All primary antibodies from Cell Signaling Technology, Inc. and Abcam were diluted at 1:1,000, while those from Santa Cruz Biotechnology, Inc. were diluted at 1:200. Primary antibodies were incubated overnight with gentle shaking at $4^{\circ} \mathrm{C}$. Subsequent to three washes with phosphate-buffered saline (PBS; Sinopharm Chemical Reagent Co., Ltd.), the membranes were then incubated with IRdye $800 \mathrm{CW}$-conjugated goat anti-rabbit immunoglobulin (Ig)G (cat. no. 926-32211; LI-COR Biosciences, Lincoln, NE, USA) and IRdye $800 \mathrm{CW}$-conjugated goat anti-mouse IgG (cat. no. 926-32210; LI-COR Biosciences) secondary antibodies at $1: 10,000$ for $1 \mathrm{~h}$ at $37^{\circ} \mathrm{C}$ in Odyssey blocking buffer (LI-COR Biosciences). The blots were scanned and analyzed using a two-color infrared imaging system (Odyssey; LI-COR Biosciences).

Cell culture. Rat H9c2 cardiac cells (Cell Bank of the Chinese Academy of Sciences, Shanghai, China) were cultured in Dulbecco's modified Eagle's medium(DMEM; cat. no. C11995;
Gibco; Thermo Fisher Scientific, Inc.), supplemented with $10 \%$ fetal bovine serum (cat. no. SV30087.02; GE Healthcare Life Sciences, Logan, UT, USA), $100 \mathrm{U} / \mathrm{ml}$ penicillin and $100 \mathrm{mg} / \mathrm{ml}$ streptomycin (cat. no. 15140; Gibco; Thermo Fisher Scientific, Inc.) in an atmosphere containing $5 \% \mathrm{CO}_{2}$ at $37^{\circ} \mathrm{C}$ in an MCO-18 $\mathrm{M}$ incubator (Panasonic, Tokyo, Japan). SSYX was dissolved in $\mathrm{dd}_{2} \mathrm{O}$ at a concentration of $10 \mathrm{mg} / \mathrm{ml}$, prior to being filtered using a $0.2 \mathrm{~mm}$ filter (cat. no. 1140503; Pall Corporation, Port Washington, NY, USA) for bacteriological sterilization. The cells were divided at 2-3-day intervals once they reached $70-80 \%$ confluence. The cells were subsequently seeded into six-well, 24-well or 96-well culture plates for $24 \mathrm{~h}$. The culture medium was then replaced with serum-free DMEM for $12 \mathrm{~h}$ prior to experimentation at $37^{\circ} \mathrm{C}$. Subsequently, the cells were incubated with $\operatorname{SSYX}(0.1,1,10$, 50 and $100 \mu \mathrm{g} / \mathrm{ml})$ at $37^{\circ} \mathrm{C}$, with or without $1 \mu \mathrm{M}$ angiotensin (Ang) II (cat. no. A9525; Sigma-Aldrich), for $24 \mathrm{~h}$. The cells were seeded at a density of $1 \times 10^{6}$ cells/well into six-well culture plates for mRNA extraction, $4 \times 10^{3}$ cells/well in 96 -well plates for cell viability assessment, $5.0 \times 10^{3}$ cells/well in 24 -well plates for cell surface area examination and $10 \times 10^{6}$ cells/well into $10 \mathrm{~cm}$ diameter culture plates for protein extraction.

Cell viability assessment. To identify whether SSYX was toxic towards the H9c2 cells, cell viability was assessed using a Cell Couting kit 8 (CCK-8) assay (cat. no. ER612; Dojindo Molecular Technologies, Inc., Kumamoto, Japan). Following treatment, $10 \mu \mathrm{l}$ CCK-8 solution at a 1/10 dilution was added to each well, and the plate was incubated at $37^{\circ} \mathrm{C}$ for $2.5 \mathrm{~h}$. The absorbance was measured at $450 \mathrm{~nm}$ using a Bio-Tek plate reader (Synergy2 model; Bio-Tek Instruments, Inc.). The means of the optical density (OD) of the five wells were used to measure the percentage of cell viability, according to the following equation: Cell viability $(\%)=\left(\mathrm{OD}_{\text {treatment }}-\mathrm{OD}_{\text {control }}\right)$ $\mathrm{x} 100 \%$. 
A
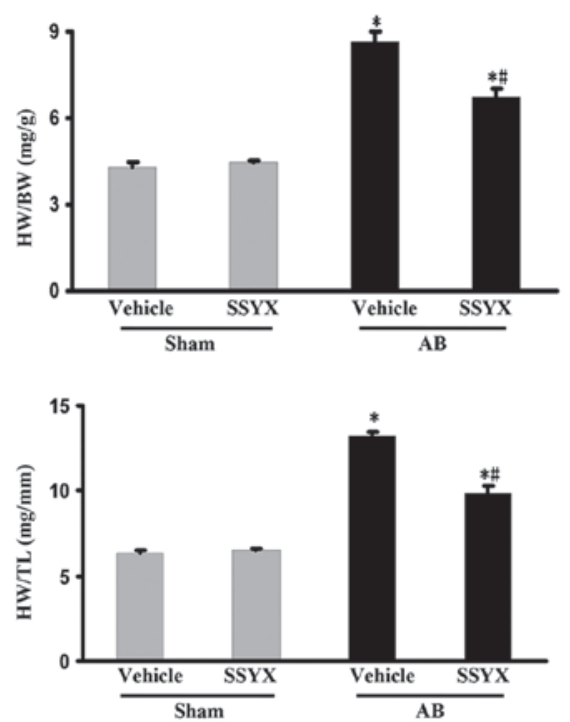
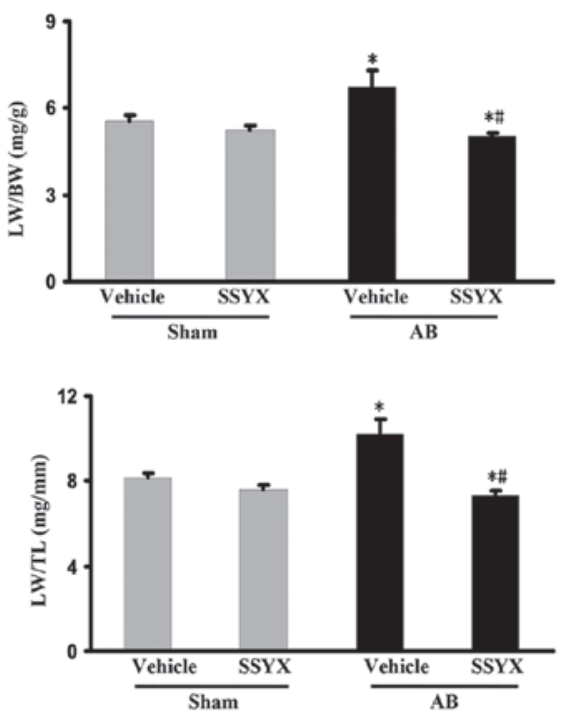

B
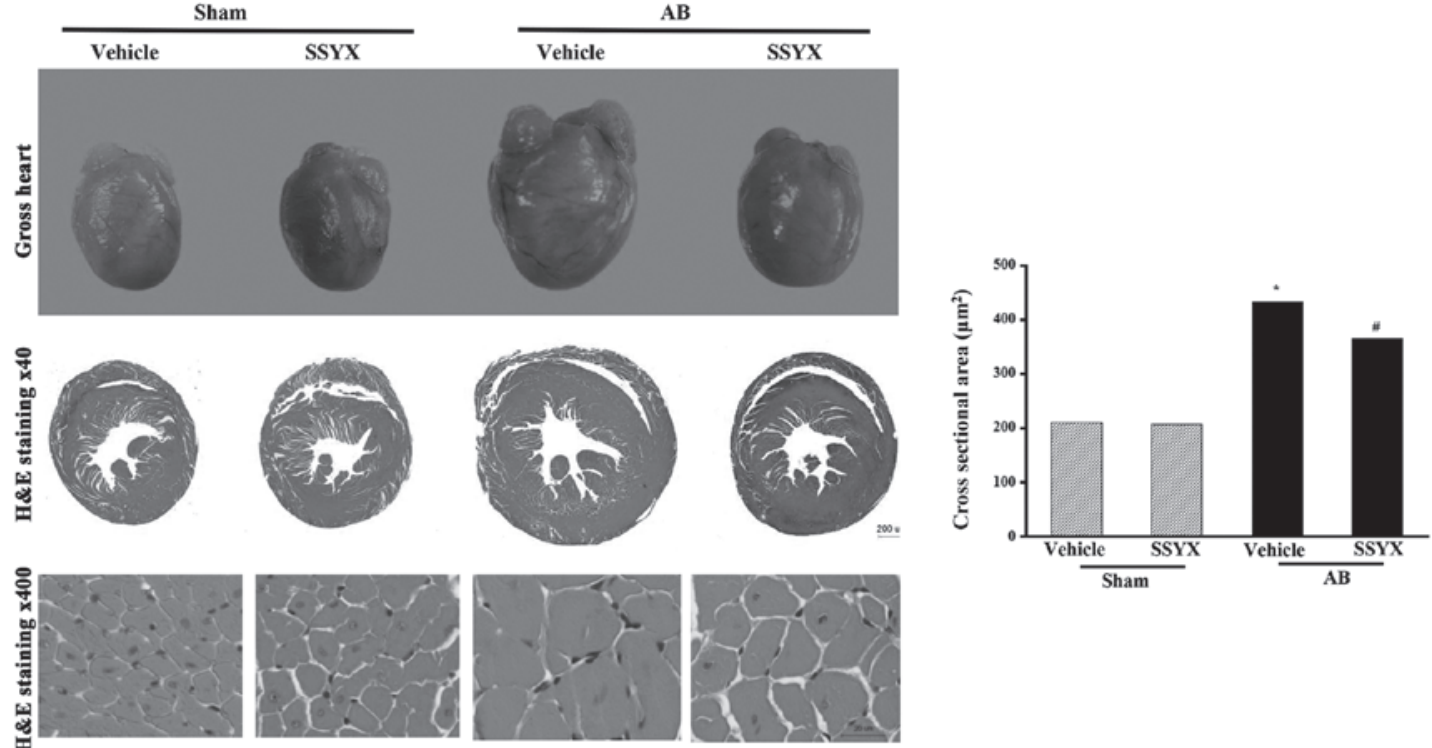

C
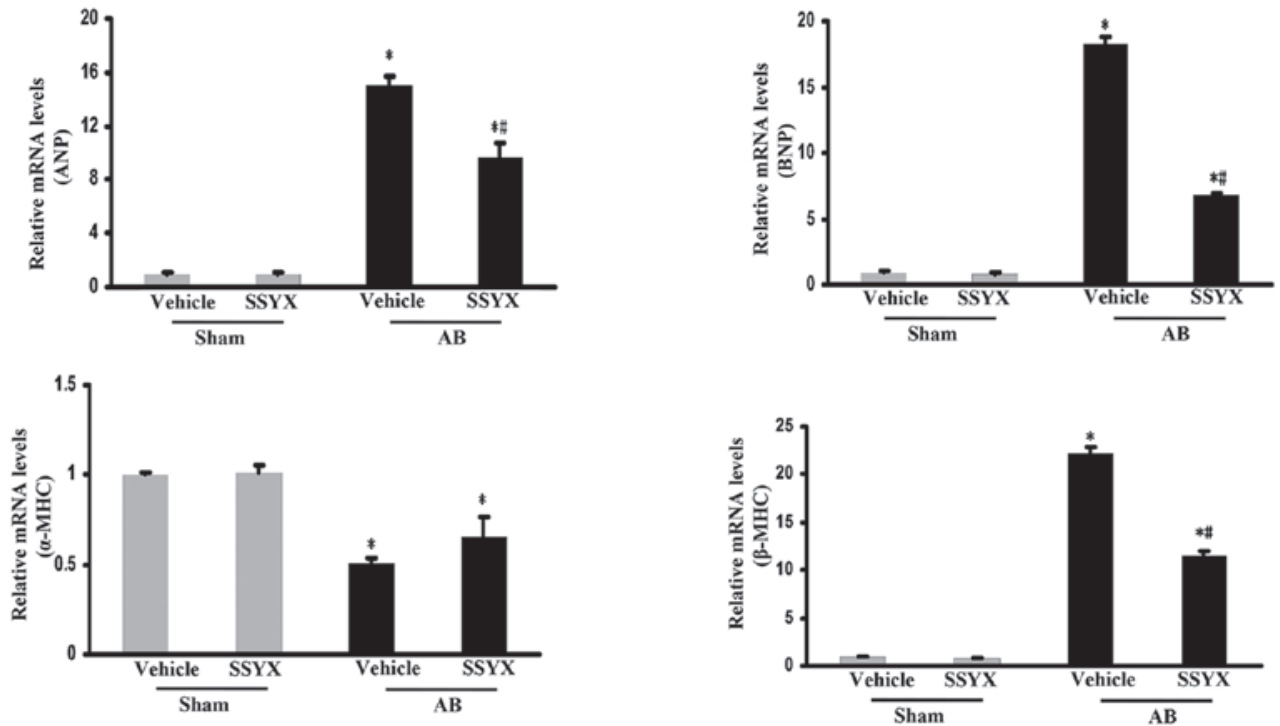

Figure 1. SSYX attenuates cardiac hypertrophy induced by pressure-overload. (A) HW/BW, LW/BW, HW/TL and LW/TL ratio of the various treatment groups. (B) Left, representative histological images of gross heart size (upper) and hematoxylin and eosin staining (middle, $\mathrm{x} 40$ magnification; bottom, $\mathrm{x} 400$ magnification) of vehicle- and SSYX-treated-mice 8 weeks following sham or AB surgery; right, statistical results for the cell surface area ( $\mathrm{n} \geq 100 \mathrm{cells})$. The image indicates that SSYX prevented increased cardiac mass and myocyte cross sectional area induced by pressure overload. (C) Reverse transcription-quantitative polymerase chain reaction analyses of the hypertrophic markers ANP, BNP, $\beta$-MHC and $\alpha$-MHC induced by AB in the mice $(n=6)$. Data are expressed as the mean \pm standard error of the mean. " $\mathrm{P}<0.05$, vs. vehicle/sham group; ${ }^{*} \mathrm{P}<0.05$, vs. vehicle/AB group. SSYX, shensongyangxin; AB, aortic banding; HW, heart weight; BW, body weight; LW, lung weight; TL, tibial length; ANP, atrial natriuretic peptide; BNP, brain natriuretic peptide; MHC, myosin heavy chain. 
A

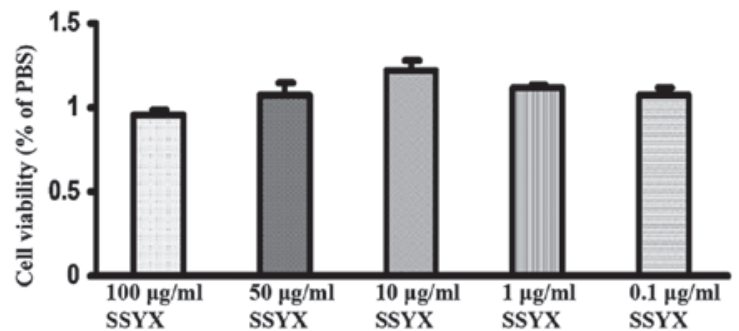

B
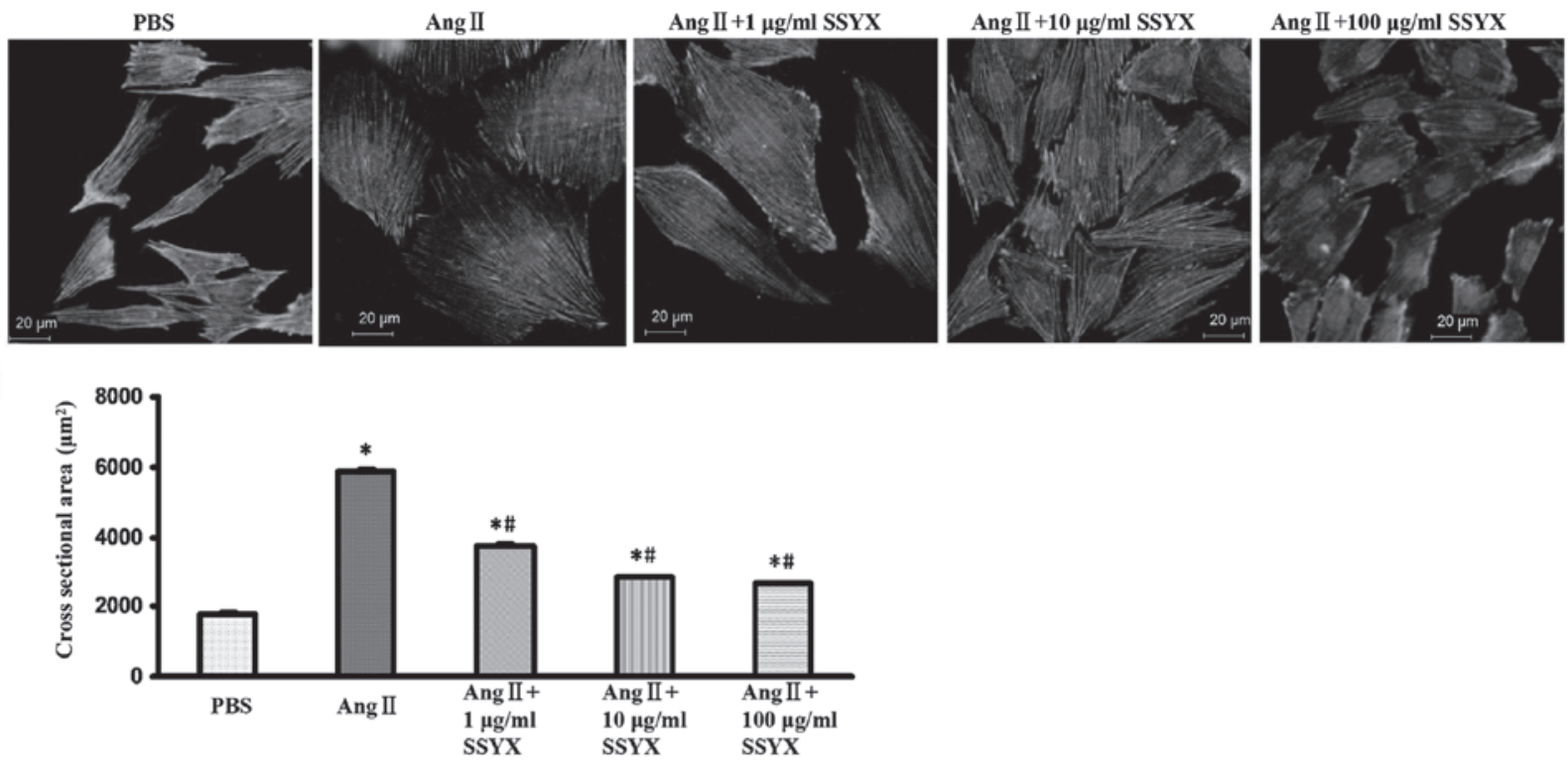

$\mathbf{C}$
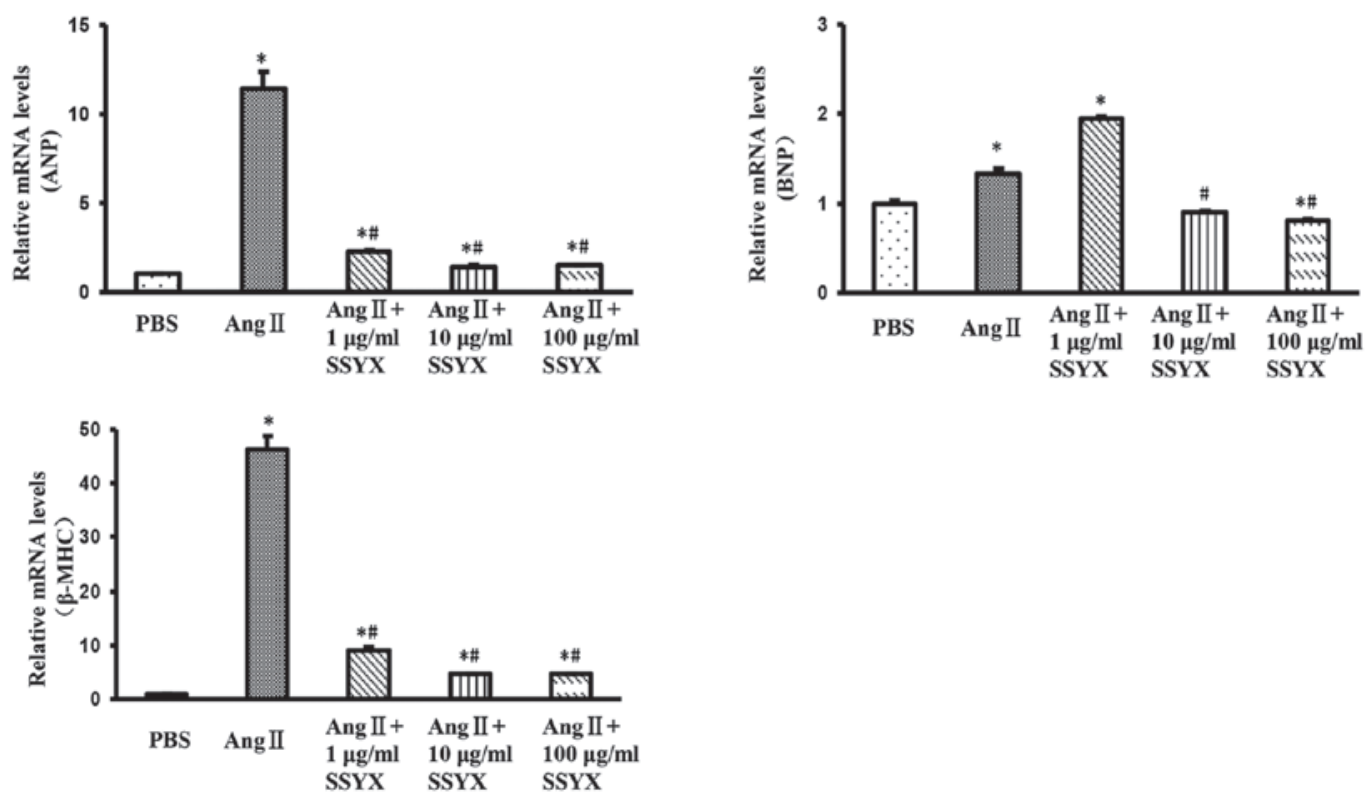

Figure 2. SSYX attenuates cell hypertrophy in vitro. (A) Cell viability following treatment with various concentrations of SSYX $(0.1,1,10,50 \mathrm{and} 100 \mu \mathrm{g} / \mathrm{ml})$. (B) Top panel, representative images of cardiomyocytes treated with various concentrations of SSYX $(1,10$ and $100 \mu \mathrm{g} / \mathrm{ml})$ in response to Ang II (1 $\mu \mathrm{M})$. Bottom panel, quantitative results of the cell surface area ( $\mathrm{n} \geq 100$ cells). The images show that SSYX $(1,10$ and $100 \mu \mathrm{g} / \mathrm{ml})$ markedly attenuated the enlarged cell surface area induced by Ang II. (C) Reverse transcription-quantitative polymerase chain reaction analysis of the mRNA expression levels of ANP, BNP and $\beta$-MHC induced by Ang II $(1 \mu \mathrm{M})$ following treatment with various concentrations of SSYX $(1,10$ and $100 \mu \mathrm{g} / \mathrm{ml})$ for $24 \mathrm{~h}$. Data are expressed as the mean \pm standard error of the mean. ${ }^{*} \mathrm{P}<0.05$, vs. PBS-treated group; ${ }^{\prime} \mathrm{P}<0.05$, vs. Ang II group. SSYX, shensongyangxin; Ang, angiotensin; ANP, atrial natriuretic peptide; BNP, brain natriuretic peptide; MHC, myosin heavy chain; PBS, phosphate-buffered saline.

Cell surface area. To identify the H9c2 cells and examine the levels of cardiomyocyte hypertrophy, the cells were characterized using immunofluorescence staining for cardiaca-actinin. Briefly, the cells were washed with PBS, fixed with RCL2 (Alphelys, Plaisir, France), permeabilized in 0.1\% Triton X-100
(Amresco LLC, Solon, OH, USA) in PBS and stained with human monoclonal anti- $\alpha$-actinin (cat. no. 05-384; Merck Millipore) at a dilution of 1:100 in 1\% goat serum overnight at $4^{\circ} \mathrm{C}$. Subsequent to 5 washes with PBS, the cells were then incubated with Alexa FluorH 568 goat anti-mouse IgG 
A
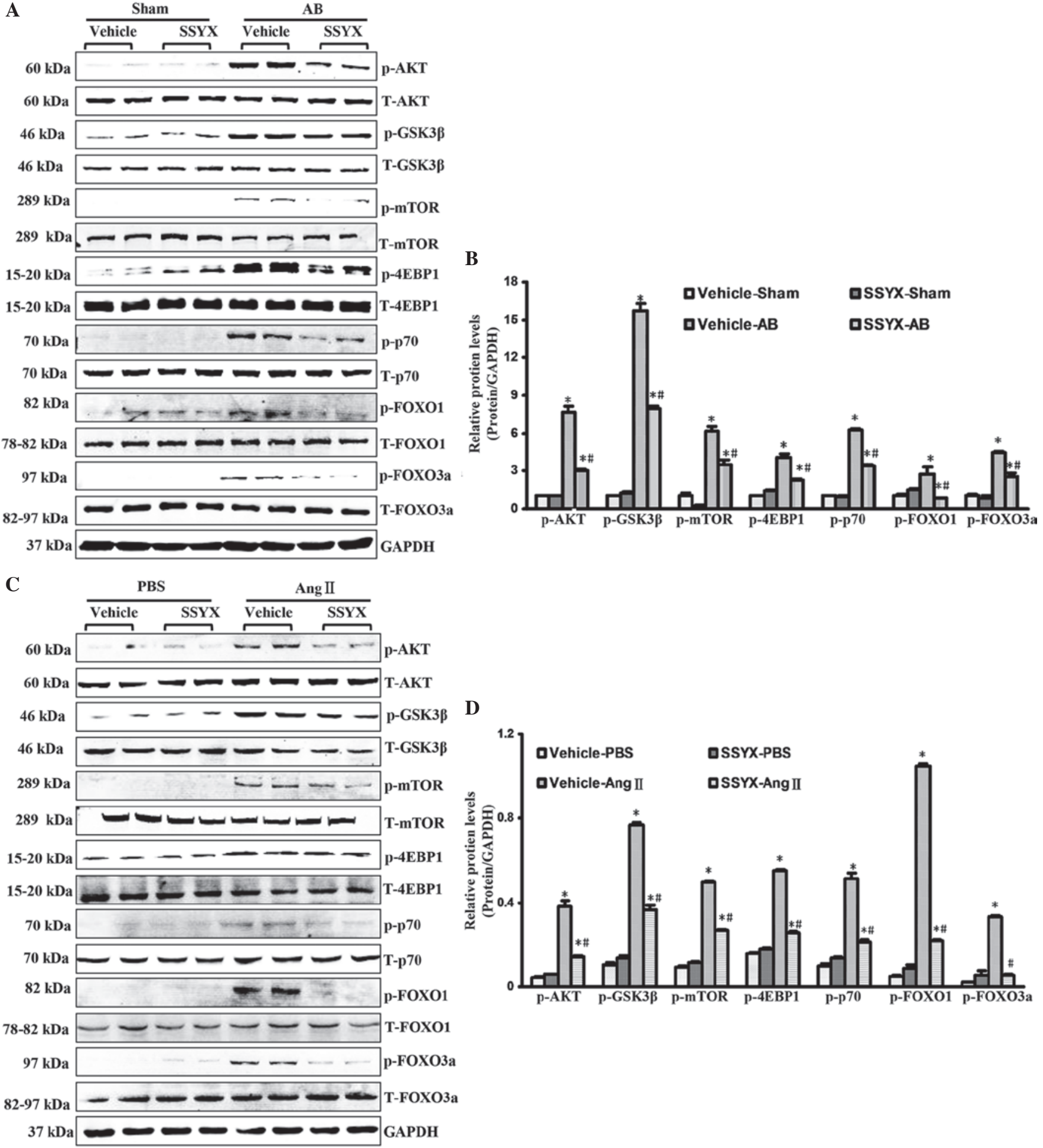

Figure 3. SSYX inhibits the activation of the phosphoinositide 3-kinase/Akt signaling pathways following AB. (A) Representative western blots of the phosphorylation and total protein expression levels of Akt, GSK3 $\beta$, mTOR, 4EBP1, p70, FOXO1 and FOXO3a in the vehicle- and SSYX-treated-mice 8 weeks following AB. (B) Quantitative results of the protein expression levels $(n=6)$. Data are expressed as the mean \pm standard error of the mean. ${ }^{*} \mathrm{P}<0.05$, vs. vehicle/sham group; ${ }^{*} \mathrm{P}<0.05$, vs. vehicle/AB group. (C) Representative western blots of the phosphorylation and total protein expression levels of Akt, GSK3 $\beta$, mTOR, 4EBP1, p70, FOXO1 and FOXo3a in the vehicle- and SSYX-treated H9c2 cardiomyocytes in response to Ang II. (D) Quantitative results of the protein expression levels $(\mathrm{n}=6)$. Data are expressed as the mean \pm standard error of the mean. $\mathrm{P}<0.05$, vs. vehicle/PBS group; ${ }^{\#} \mathrm{P}<0.05$, vs. vehicle/Ang II group. SSYX, shensongyangxin; $\mathrm{AB}$, aortic banding; PBS, phosphate-buffered saline; GSK3 $\beta$, glycogen synthase kinase 3 $\beta$; mTOR, mammalian target of rapamycin; 4EBP1, $4 \mathrm{E}$ binding protein 1; FOX, forkhead box protein; T-, total; p-, phosphorylated.

monoclonal secondary antibody (cat. no. A11004; Invitrogen; Thermo Fisher Scientific, Inc.) for $60 \mathrm{~min}$ at room temperature. Following 6 washes with PBS, the cells on coverslips were mounted onto glass slides using SlowFade Gold antifade reagent with DAPI (cat. no. S36939; Invitrogen; Thermo Fisher Scientific, Inc.). A single cell was measured using the Image Pro-Plus 6.0 quantitative digital image analysis system. The outline of 40 cells was traced for each group. 
A

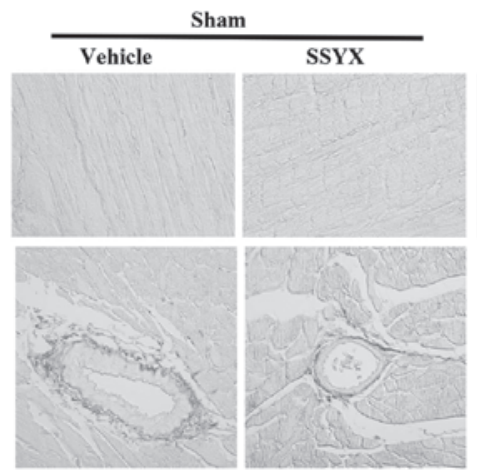

C
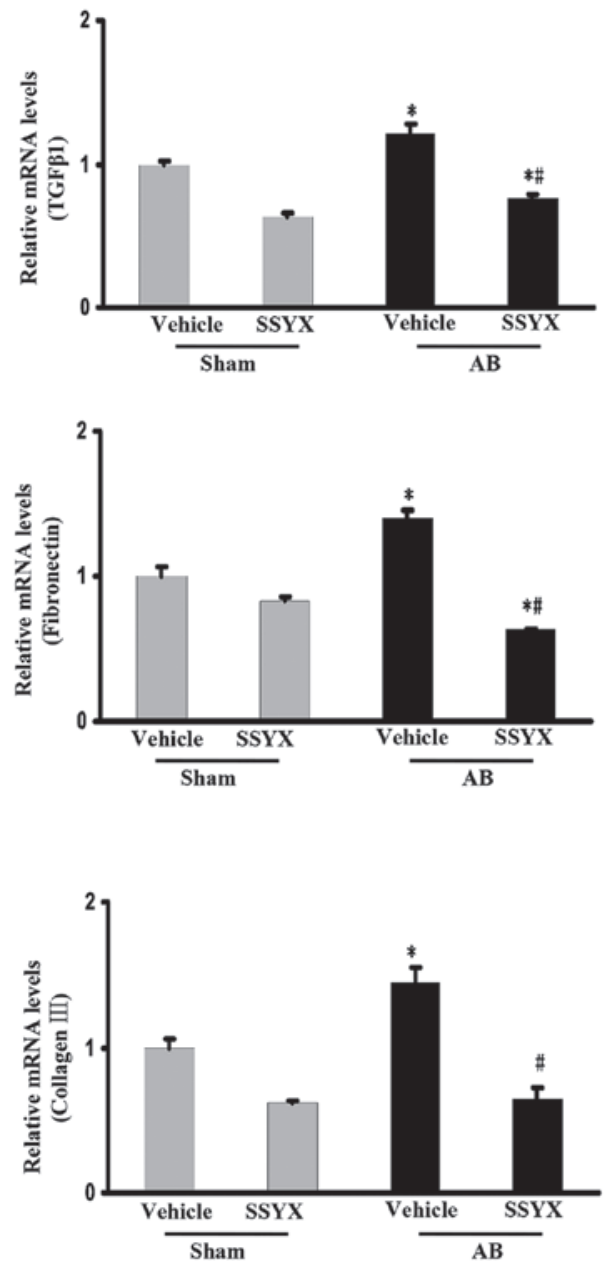

B
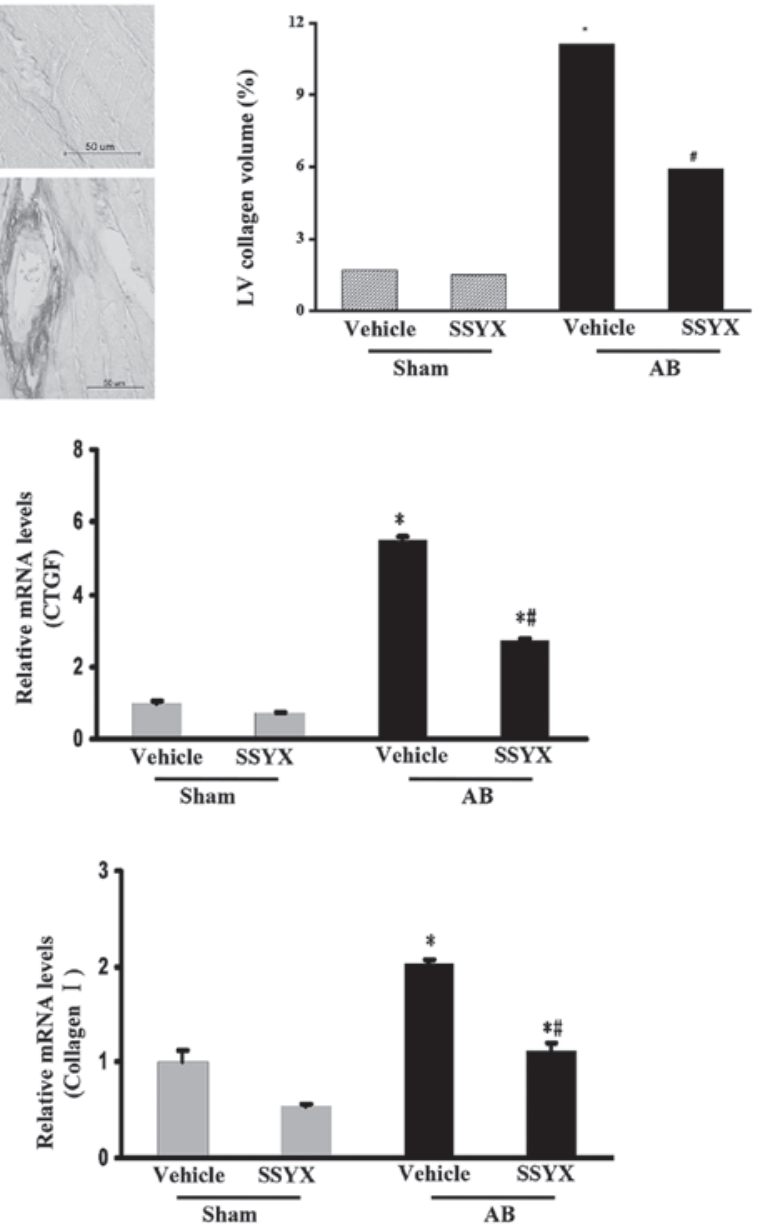

D
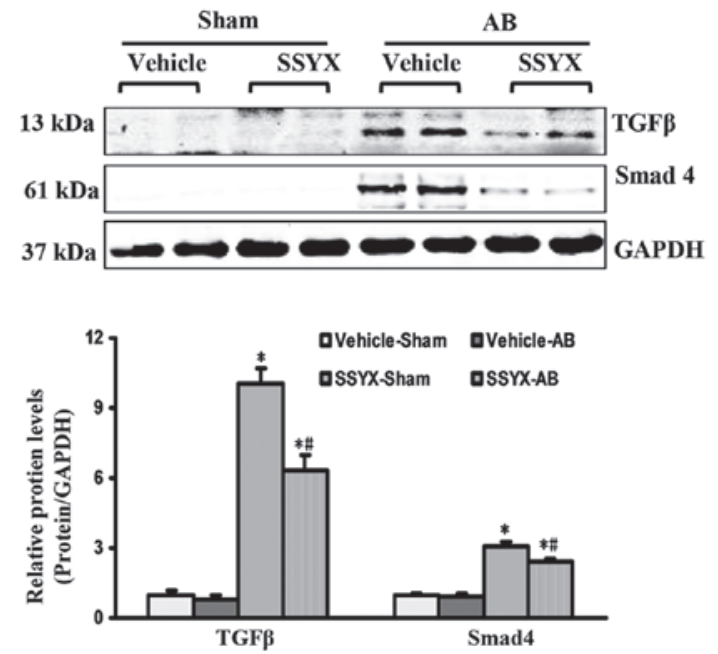

Figure 4. SSYX inhibits cardiac fibrosis induced by pressure overlord. (A) Picrosirius red staining of the histological sections of the left ventricule in the various treatment groups eight weeks following $\mathrm{AB}(\mathrm{n}=6$; scale bar, $50 \mu \mathrm{m})$. Upper, interstitial fibrosis (magnification, $\mathrm{x} 400)$; bottom, perivascular fibrosis (magnification, x200). The images show that the extent of cardiac fibrosis was significantly reduced in the SSYX-treated mice. (B) Quantitative results of the expression levels of the proteins. (C) Reverse transcription-quantitative polymerase chain reaction analysis of the mRNA expression levels of TGF $\beta 1$, CTGF, and fibrotic markers fibronectin, collagen I $\alpha$ and collagen III in the mice (n=6). (D) Representative western blots demonstrating the protein expression of TGF $\beta$ and Smad4. Top panel: Representative western blots; bottom panel: Quantitative results $(n=6) .{ }^{~} \mathrm{P}<0.05$, vs. the vehicle/sham group; ${ }^{*} \mathrm{P}<0.05$, vs. the vehicle/AB group. SSYX, shensongyangxin; AB, aortic banding; TGF, transforming growth factor; CTGF, connective tissue growth factor.

Statistical analysis. Statistical analysis was conducted using SPSS software, version 13.0 (SPSS, Inc., Chicago, IL, USA). Data are expressed as the mean \pm standard error of the mean.
Statistical differences among the treatment groups were determined using one-way analysis of variance followed by a Tukey's post-hoc test. Comparisons between two groups 
were performed using unpaired Student's t-test. $\mathrm{P}<0.05$ was considered to considered to indicate a statistically significant difference.

\section{Results}

SSYX attenuates pressure overload-induced cardiac hypertrophy. To investigate whether SSYX has an antagonistic effect on the hypertrophic response to pressure overload, mice were subjected to pressure load via AB or sham surgery. SSYX prevented the development of adverse cardiac remodeling and ventricular dysfunction, as demonstrated by the HW/BW, HW/TL, LW/BW and LW/TL ratios (Fig. 1A), and increased cardiac mass and myocyte cross sectional area (Fig. 1B). The expression levels of ANP, BNP and $\beta$-myosin heavy chain (MHC) hypertrophic markers following AB surgery were also significantly decreased in the SSYX-treated mice, whereas the level of $\alpha$-MHC was significantly increased in the SSYX-treated mice following AB (Fig. 1C). These results suggested that SSYX negatively regulated the level of cardiac hypertrophy in response to the pressure overload.

SSYX attenuates cell hypertrophy in vitro. To further validate the effect of SSYX on cardiac hypertrophy, an Ang II $(1 \mu \mathrm{M})$ in vitro model was used in cultured $\mathrm{H} 9 \mathrm{c} 2$ cells. The cytotoxicity of SSYX was assessed in the absence of Ang II using a CCK-8 assay. No differences were observed between the viabilities of the H9c2 cardiomyocytes treated with different concentrations of $\operatorname{SSYX}(0.1,1,10,50$ and $100 \mu \mathrm{g} / \mathrm{ml})$ and vehicle control group (Fig. 2A). These results suggested that SSYX did not exert a cytotoxic effect on the H9c2 cardiomyocytes, and further experiments were performed. Following stimulation with Ang II $(1 \mu \mathrm{M})$, the H9c2 cells exhibited enlarged cell surface areas, compared with control group, and SSYX $(1,10$ and $100 \mu \mathrm{g} / \mathrm{ml})$ markedly attenuated this enlarged cell surface area (Fig. 2B). The results of the RT-qPCR analysis demonstrated that treatment with $\operatorname{SSYX}(1,10$ and $100 \mu \mathrm{g} / \mathrm{ml})$ markedly decreased the Ang II $(1 \mu \mathrm{M})$-induced mRNA expression levels of ANP, BNP and $\beta$-MHC, particularly following treatment with 10 and $100 \mu \mathrm{g} / \mathrm{ml} \mathrm{SSYX} \mathrm{(Fig.} \mathrm{2C).} \mathrm{These} \mathrm{find-}$ ings were concordant with the in vivo results, and suggested that SSYX attenuated cardiac hypertrophy.

SSYX inhibits activation of the Akt signaling pathway following $A B$. To investigate the molecular mechanisms underlying the antihypertrophic effects of SSYX, activation of the Akt signaling pathway was examined. Pressure overload led to increases in the phosphorylation of Akt, mTOR, GSK3, 4EBP1, p70, FOXO1 and FOXO3a 8 weeks following surgery. Notably, SSYX significantly attenuated these increased phosphorylation levels, as shown in Fig. 3A and 3B. The in vitro data confirmed that the phosphorylation levels of Akt, mTOR, GSK3, 4EBP1, p70, FOXO1 and FOXO3a in the H9c2 cells following treatment with $\operatorname{SSYX}(10 \mu \mathrm{M})$ were lower, thanthose in the cells treated with $1 \mu \mathrm{M}$ Ang II (Fig. 3C and D). These results demonstrated that SSYX significantly inhibited cardiac hypertrophy via inhibition of the Akt signaling pathway.

SSYX inhibits pressure overload-induced cardiac fibrosis. To evaluate the levels of fibrosis in the heart following exposure to SSYX, the paraffin-embedded slides were stained with PSR. Perivascular and interstitial fibrosis were observed in the vehicle and SSYX-treated mice subjected to AB, however, the extent of cardiac fibrosis was significantly reduced in the SSYX-treated mice (Fig. 4A and B). The expression levels of myocardial pro-fibrotic genes 8 weeks following surgery were also examined. As shown in Fig. $4 \mathrm{C}$, the pressure overload-induced expression of TGF $\beta 1$, CTGF, fibronectin, collagen I and collagen III were decreased by SSYX. Therefore, the present study investigated the TGF $\beta / S m a d$ signaling pathway. The expression levels of TGF $\beta$ and Smad4 were increased by pressure overload, and treatment with SSYX significantly decreased these expression levels (Fig. 4D). These results indicated that SSYX significantly attenuated cardiac fibrosis through inhibition of the TGF $\beta /$ Smad signaling pathway.

\section{Discussion}

SSYX is a compound of the traditional Chinese Materia medica, consisting of 12 ingredients, and has attracted considerable attention due to its anti-arrhythmic properties (9-11). Previous studies have suggested that SSYX exhibits protective effects on the cardiovascular system $(12,13)$. However, the role of SSYX in pressure overload-induced cardiac remodeling remains to be elucidated. The present study demonstrated that treatment with SSYX attenuated the remodeling process of the heart in response to pressure overload, including cardiac hypertrophy and interstitial fibrosis. In addition, treatment with SSYX attenuated cardiomyocyte hypertrophy induced by Ang II in vitro. To the best of our knowledge, the present study is the first to demonstrate the important role of SSYX in the regulation of cardiac hypertrophy and fibrosis.

Cardiac hypertrophy, which is observed in a wide variety of clinical conditions, is a major pathological process of heart failure (7). Several investigations have been performed to elucidate the mechanisms that stimulate or prevent hypertrophy, identifying a variety of signaling pathways and transcription factors, however, these mechanisms remain to be fully elucidated $(16,17)$. Akt is important in cardiac hypertrophy of the tyrosine kinase receptor signaling pathways, which regulate cell morphology, cell survival, angiogenesis and inflammation in cardiomyocytes $(5,18)$. Cardiac-specific overexpression of a constitutively active form of Akt leads to significant cardiac hypertrophy, which may be due to increased cardiomyocyte size (16). GSK3 3 , which is located downstream of Akt, is inactivated by the phosphorylation of serine 9 under hypertrophic conditions, and has been shown to be a negative regulator of cardiomyocyte hypertrophy $(14,19)$. Among the downstream effectors of Akt, mTOR is activated by Akt phosphorylation (16). mTORC1, a complex form of mTOR, stimulates protein synthesis through the eukaryotic initiation factor, 4E-BP1, and p70S6 kinase during protein translation, and the acceleration of protein translation can enhance cell growth and mass $(16,20)$. To examine the molecular mechanisms underlying the protective effects of SSYX against cardiac hypertrophy, the activity of Akt signaling was examined in the hypertrophic models. The results demonstrated that Akt signaling activation was inhibited in the SSYX-treated hearts and H9c2 cells in response to chronic pressure overload and stimulation with Ang II. 
The forkhead box O (FOXO) transcription factor, a downstream target of the Akt signaling pathway, comprises three members: FOXO1 (FKHR), FOXO3a (FKHRL-1) and FOXO4 (AFX), all of which are inactivated by Akt (17). Phosphorylation by Akt leads to nuclear exclusion and inhibits the forkhead transcriptional program (17). FOXO transcription factors regulate key physiological functions, including responses to stress, cell-cycle progression, protein degradation and apoptosis (17). A previous study demonstrated that overexpression of either FOXO1 or FOXO3 decreased calcineurin phosphatase activity, and hearts from FOXO3-null mice manifest a hypertrophic phenotype (21). Therefore, the effects of SSYX on the expression levels of FOXO1 and FOXO3a were further examined in vivo and in vitro in the present study. The findings indicated that the inhibitory effects of SSYX on cardiac hypertrophy were mediated through Akt signaling.

Cardiac fibrosis is an important hallmark of maladaptive hypertrophy and heart failure, and is characterized by an increase in the levels of collagen and other extracellular matrix (ECM) components in the interstitium and perivascular regions of the myocardium (6). TGF $\beta 1$ is an important regulator of ECM metabolism in various organs (22). ECM production in the heart is regulated by TGF $\beta$. Canonical signaling via TGF $\beta$ receptors activates Smad proteins, which phosphorylate Smad2/3 on two serine residues at their C-terminus and allows binding to Smad4 to form heteromeric Smad complexes. These enter the nucleus to initiate gene transcription (23). An important profibrotic target gene of TGF $\beta$ signaling is CTGF, which is essential for TGF $\beta$-induced collagen synthesis (24). The antifibrotic effects of SSYX were demonstrated in the present study by the significant decreases in the mRNA expression levels of TGF $\beta 1$, CTGF, and ECM proteins, including collagen Ia, collagen III and fibronectin in the SSYX-treated mice. The expression levels of TGF $\beta 1$ and Smad4 were also decreased, suggesting that activation of the TGF $\beta 1 /$ Smad signaling pathway following $\mathrm{AB}$ was attenuated by SSYX, which may mediate the antifibrotic effects of SSYX.

In conclusion, the present study demonstrated that SSYX exerted protective effects against cardiac hypertrophy and fibrosis in response to chronic pressure overload and Ang II stimulation by regulating the Akt and TGF $\beta 1 /$ Smad signaling pathways. These results provide experimental evidence for the potential application of SSYX in the treatment of cardiac remodeling and $\mathrm{HF}$.

\section{Acknowledgements}

The current study was supported by the National Key Basic Research Program of China (973 Program; grant no. 2012CB518606) and grants from the Fundamental Research Funds for the Central Universities of China (grant no. 2014302020202).

\section{References}

1. Rosca MG, Tandler B and Hoppel CL: Mitochondria in cardiac hypertrophy and heart failure. J Mol Cell Cardiol 55: 31-41, 2013

2. Foryst-Ludwig A and Kintscher U: Sex differences in exercise-induced cardiac hypertrophy. Pflugers Arch 465: 731-737, 2013.
3. Abel ED and Doenst T: Mitochondrial adaptations to physiological vs. pathological cardiac hypertrophy. Cardiovasc Res 90: 234-242, 2011.

4. Hou J and Kang Y: Regression of Pathological Cardiac Hypertrophy: Signaling pathways and therapeutic targets. Pharmacol Ther 135: 337-354, 2012.

5. Pillai VB, Sundaresan N and Gupta MP: Regulation of Akt signaling by sirtuins: Its implication in cardiac hypertrophy and aging. Circ Res 114: 368-378, 2014.

6. Creemers EE and Pinto Y: Molecular mechanisms that control interstitial fibrosis in the pressure-overloaded heart. Cardiovasc Res 89: 265-272, 2011.

7. Oka T, Akazawa H, Naito AT and Komuro I: Angiogenesis and cardiac hypertrophy: Maintenance of cardiac function and causative roles in heart failure. Circ Res 114: 565-571, 2014.

8. Liu Z HJ, Hu R, Huo Y, Gong J, Zhang Y, Wei C and $\mathrm{Pu} \mathrm{J}$ : Gene expression profile of increased heart rate in shensongyangxin-treated bradycardia rabbits. Evid Based Complement Alternat Med 2014: 715937, 2014

9. Liu Y, Li N, Jia Z, Lu F and Pu J: Chinese medicine shensongyangxin is effective for patients with bradycardia: Results of a randomized, double-blind, placebo-controlled multicenter trial. Evid Based Complement Alternat Med 2014: 605714, 2014.

10. Wang AH, Pu JL, Qi XY, Miao WL, Hou ZS, Cong HL, Zhou JZ, Liu XF, Li SM and Han QH: Evaluation of shensongyangxin capsules in the treatment of paroxysmal atrial fibrillation: A randomized, double-blind and controlled multicenter trial. Zhonghua Yi Xue Za Zhi 91: 1677-1681, 2011 (In Chinese).

11. Zou JG, Zhang J, Jia ZH and Cao KJ: Evaluation of the traditional Chinese Medicine Shensongyangxin capsule on treating premature ventricular contractions: A randomized, double-blind, controlled multicenter trial. Chin Med J (Engl) 124: 76-83, 2011.

12. Yang Z, Yu X and Yu ML: Effects of shensongyangxin capsule on heart rate turbulence and heart rate variability in chronic heart failure. Chin Med J (Engl) 126: 4389-4391, 2013.

13. Chai S, Wang S, Yao L, Wu A, Liu Y and Rao C: Effect of shensongyangxin capsule on myocardial remodeling and ventricular fibrillation threshold value in rat with coronary artery ligation. Zhongguo Zhong Yao Za Zhi 34: 2101-2104, 2009 (In Chinese).

14. Yuan Y Zong J, Zhou H, Bian ZY, Deng W, Dai J, Gan HW, Yang Z, Li H and Tang QZ: Puerarin attenuates pressure overload-induced cardiac hypertrophy. J Cardiol 63: 73-81, 2014.

15. Yan L, Wei X, Tang QZ, Feng J, Zhang Y, Liu C, Bian ZY, Zhang LF, Chen M, Bai X, et al: Cardiac-specific mindin overexpression attenuates cardiac hypertrophy via blocking AKT/GSK3 $\beta$ and TGF- $\beta 1-$ Smad signalling. Cardiovasc Res 92: 85-94, 2011.

16. Aoyagi T and Matsui T: Phosphoinositide- 3 kinase signaling in cardiac hypertrophy and heart failure. Curr Pharm Des 17: 1818-1824, 2011.

17. Skurk C, Izymiya Y, Maatz H, Razeghi P, Shiojima I, Sandri M, Sato K, Zeng L, Schiekofer S, Pimentel D, et al: The FOXO3a transcription factor regulates cardiac myocyte size downstream of AKT signaling. J Biol Chem 280: 20814-20823, 2005.

18. Yu W, Chen C, Fu Y, Wang X and Wang W: Insulin signaling: A possible pathogenesis of cardiac hypertrophy. Cardiovasc Ther 28: 101-105, 2010.

19. Wu QQ, Zonj J, Gao L, Dai J, Yang Z, Xu M, Fang Y, Ma ZG and Tang QZ: Sulforaphane protects H9c2 cardiomyocytes from angiotensin II-induced hypertrophy. Herz 39: 390-396, 2014.

20. Maillet M, van Berlo JH and Molkentin JD: Molecular basis of physiological heart growth: Fundamental concepts and new players. Nat Rev Mol Cell Biol 14: 38-48, 2013.

21. Ni YG, Berenji K, Wang N, Oh M, Sachan N, Dey A, Cheng J, Lu G, Morris DJ, Castrillon DH, et al: FOXo transcription factors blunt cardiac hypertrophy by inhibiting calcineurin signaling. Circulation 114: 1159-1168, 2006.

22. Edgley AJ, Krum H and Kelly DJ: Targeting fibrosis for the treatment of heart failure: A role for transforming growth factor- $\beta$. Cardiovasc Ther 30: e30-40, 2014.

23. Kamato D, Burch M, Piva TJ, Rezaei HB, Rostam MA, Xu S, Zheng W, Little PJ and Osman N: Transforming growth factor- $\beta$ signalling: Role and consequences of Smad linker region phosphorylation. Cell Signal 25: 2017-2024, 2013.

24. Szabó Z, Magga J, Alakoski T, Ulvila J, Piuhola J, Vainio L, Kivirikko KI, Vuolteenaho O, Ruskoaho H, Lipson KE, et al: Connective tissue growth factor inhibition attenuates left ventricular remodeling and dysfunction in pressure overload-induced heart failure. Hypertension 63: 1235-1240, 2014. 